\title{
Vorwort der Autor(inn)en
}

Behinderte Kinder - behinderte Eltern, so bringen Familien ihre Erfahrungen häufig auf den Punkt. „Auch wenn unser Kind behindert ist, sind wir als Familie, bin ich als Vater noch lange nicht behindert“, sagte ein Vater in einem Gespräch. Er möchte seine Vaterschaft genauso leben wie andere Väter auch, dabei engagiert er sich weitaus stärker in seiner Familie als noch sein eigener Vater, so wie die meisten anderen Väter heute auch. Väter von Kindern mit Behinderung übernehmen Fürsorge- und Pflegeaufgaben und möchten mit ihren Kindern mehr Zeit verbringen, mit ihnen Spaß haben und ganz normale Dinge tun. Dabei fühlen sie sich von der Gesellschaft behindert, da es auch zehn Jahre nach Unterzeichnung der Behindertenrechtskonvention noch lange nicht selbstverständlich ist, dass sie mit ihrem behinderten Kind teilhaben können und dazugehören. Sie stehen vor Barrieren, ernten mitleidige Blicke, treffen auf Sprachlosigkeit oder Vorsicht bei ihrem Gegenüber, nur nichts Falsches zu sagen. Obwohl sie ganz normal sein möchten, befinden sie sich in einer besonderen Situation mit spezifischen Herausforderungen, Fragen und Problemen. Die Einrichtungen, die ihre Kinder betreuen, bieten Eltern Gespräche dafür an und laden Väter ein, mitzukommen. Aber auch hier fühlen sich Väter nicht wirklich zugehörig, da diese Angebote häufig nicht ihren Bedürfnissen entsprechen.

Wir schätzen es sehr, dass sich das Bayerische Staatsministerium für Familie, Arbeit und Soziales im Jahr 2012 mit der Förderung des Praxis- wie auch Begleitforschungsprojekts „Arbeit mit Vätern von Kindern mit Behinderung“ diesem hoch aktuellen und hoch politischen Thema zugewandt hat und nun auch die Väter von Kindern mit Behinderung Aufmerksamkeit und Unterstützung erfahren. Denn obwohl es bislang wenig Untersuchungen dazu gibt, sind wir fest überzeugt, und sehen das auch an den Befunden aus unserem Projekt, dass sich in Familien mit einem Kind mit Behinderung Verschiebungen in den Zuständigkeiten ergeben und damit auch in den Aufgaben und Rollen von Vätern. Und wir sehen, dass die Angebote, die in den letzten Jahren im Rahmen dieses Projekts an mehreren Standorten entwickelt wurden, ebenfalls zu einer Veränderung und Stärkung der Vaterrolle beitragen können. Damit ermöglichen sie einen ersten kleinen Schritt zu mehr Teilhabe für Väter und ihre Kinder auch in anderen gesellschaftlichen Bereichen.

Mit dem Titel „Väter an Bord“ wollten wir unsere Erfahrungen mit den Angeboten und der Teilnahme der Väter in doppeltem Sinne ausdrücken. Denn zum einen ist es im übertragenen Sinne gelungen, Väter an Bord zu holen und ihnen auch mehr und mehr das Steuer zu überlassen, und zum anderen war ein nicht geringer Teil der Angebote eine Bootsfahrt bzw. Raftingtour. Wie in langjährigen Projekten üblich, sind wir mit unseren Befunden an die Fachöffentlichkeit gegangen, konnten auf 
Kongressen und Konferenzen engagierte Diskussionen anregen und mit Zeitschriften und Aufsätzen auf dieses wichtige Thema aufmerksam machen. Für das Bayerische Staatsministerium für Familie, Arbeit und Soziales konnten wir eine Handreichung erstellen. Mit dieser Veröffentlichung ist es uns nun möglich, einen umfangreichen, differenzierten und aktuellen Beitrag zu Vätern von Kindern mit Behinderung, aber auch zu Vätern ganz allgemein zu verfassen.

Dieses Buch ist Produkt einer engen Verzahnung von Praxis und Forschung, so war auch das Projekt von Anfang an angelegt. Und wie es in einem Projektzusammenhang üblich ist, waren viele Personen daran beteiligt. Den Anstoß dazu gab Peter Barbian, Geschäftsführer der Bildungs- und Erholungsstätte Langau e. V., der 2012 das Praxisprojekt beantragt hat und dabei auch die Zusage für eine Förderung der Begleitforschung erreichen konnte. Das Projekt wurde in Kooperation mit Einrichtungen an unterschiedlichen Standorten durchgeführt. Ohne die konstruktive Zusammenarbeit mit den Kolleg(inn)en vor Ort, ihr Engagement sowie die Offenheit, mit der sie uns Auskunft gaben und einen Einblick in ihren beruflichen Alltag gewährten, wäre das Projekt nicht durchführbar gewesen. Am meisten beeindruckten uns jedoch die Väter und Familien, die an den Veranstaltungen teilnahmen und uns in der Begleitforschung teilnehmen ließen, sich unseren Fragen stellten sowie in den Interviews aufschlussreiche und spannende, teilweise auch sehr private und berührende Auskünfte zu ihrer Situation, ihren Wünschen und Fragen gegeben haben.

Darüber hinaus waren eine Reihe von Personen direkt oder indirekt am Entstehen dieses Buches beteiligt, Expert(inn)en, die mit uns über ihre Erfahrungen mit und Einschätzungen zur Männer-/Väterarbeit gesprochen haben, unsere Kolleg(inn)en, die uns durch ihre Fragen angeregt und mit uns diskutiert haben sowie Veronika Beyermann, Katja Bugelnig, Regina Kirchschlager und Franziska Harbich, die wertvolle Zuarbeiten geleistet haben. Unterstützend waren auch Kolleg(inn)en aus der Gleichstellungsarbeit sowie Einrichtungen für Menschen mit Behinderung, die das Projekt wohlwollend begleitet, hilfreiche Anregungen gegeben und nicht zuletzt auch viel für die Angebote geworben haben. Einen wichtigen Beitrag leistete schließlich auch unsere Lektorin Rita Güther, die dem Buch den letzten Schliff gegeben hat.

Neben allen diesen finanziellen, fachlichen und sozialen Ressourcen wäre die Arbeit an diesem Buch ohne die Unterstützung unserer Familien nicht möglich gewesen, die manche Wochenenden und Abende toleriert haben, die wir am Schreibtisch gesessen sind.

Besonders freut uns, dass die Arbeit mit und zu den Vätern eine nachhaltige Wirkung entfalten konnte und eine Fachstelle „Väter von Kindern mit Behinderung“ an der Bildungs- und Erholungsstätte Langau durch das Bayerische Staatsministerium für Familie, Arbeit und Soziales weiterfinanziert wurde, um die Fachöffentlichkeit auf Väterarbeit aufmerksam machen und Einrichtungen in ihrer Väterarbeit unterstützen 
und begleiten zu können. In allen Projektphasen haben wir die zuständigen Personen nicht nur als „Ernährer/-innen“ des Projekts erlebt, sondern immer an Inhalten und Weiterentwicklung Interessierte.

Ihnen allen gilt unser herzlicher Dank!

Benediktbeuern, München, Langau, Mai 2019

Luise Behringer

Wolfgang Gmür

Gerhard Hackenschmied

Daniel Wilms 